\title{
SOLITON DYNAMICS IN A POTENTIAL
}

\author{
J. C. Bronski And R. L. Jerrard
}

\begin{abstract}
We study the semiclassical limit of subcritical focussing NLS with a potential, for initial data of the form $s\left(\frac{x-x_{0}}{\epsilon}\right) e^{i \frac{v_{0} \cdot x}{\epsilon}}$, where $s$ is the ground state of an associated unscaled problem. We show that in the semiclassical limit, the solution has roughly the form $s\left(\frac{x-x^{\epsilon}(t)}{\epsilon}\right) e^{i \frac{v^{\epsilon}(t) \cdot x}{\epsilon}}$, and we show that the approximate center of mass $x^{\epsilon}(\cdot)$ converges to a solution of the equation $x^{\prime \prime}=-D V(x), x(0)=x_{0}, x^{\prime}(0)=v_{0}$ as $\epsilon \rightarrow 0$.
\end{abstract}

\section{Introduction}

In this paper we study the $\epsilon \rightarrow 0$ limit of solutions $u^{\epsilon}: \mathbb{R}^{n} \times[0, \infty) \rightarrow \mathbb{C} \cong \mathbb{R}^{2}$ of the equation

$$
i u_{t}^{\epsilon}+\frac{\epsilon}{2} \Delta u^{\epsilon}+\frac{1}{\epsilon}\left|u^{\epsilon}\right|^{p-1} u^{\epsilon}-\frac{1}{\epsilon} V(x) u^{\epsilon}=0
$$

with initial data

$$
u^{\epsilon}(\cdot, 0)=\phi^{\epsilon}, \quad \text { where } \phi^{\epsilon}(x) \sim s\left(\frac{x-x_{0}}{\epsilon}\right) e^{i \frac{v_{0} \cdot x}{\epsilon}},
$$

where $s$ is the ground state solution of an associated scalar elliptic problem. This data can be thought of as corresponding to a point particle with position $x_{0}$ and velocity $v_{0}$. Roughly speaking, we show that in the limit $\epsilon \rightarrow 0, u^{\epsilon}(x, t) \sim$ $\rho^{\epsilon} e^{i \frac{v^{\epsilon}(t) \cdot x}{\epsilon}}$, where $\rho^{\epsilon}(x, t) \sim s\left(\frac{x-X^{\epsilon}(t)}{\epsilon}\right)$, the center of mass $X^{\epsilon}(t)$ of the solution converges, as $\epsilon \rightarrow 0$ to the solution of the ordinary differential equation

$$
x^{\prime \prime}=-D V(x), \quad x(0)=x_{0}, x^{\prime}(0)=v_{0},
$$

and $v^{\epsilon}(t) \rightarrow v(t)=x^{\prime}(t)$.

Similar problems in linear geometric asymptotics were studied intensively in the 70's, see for example Guilleman and Sternberg [7]. Motivated by these linear results, Alan Weinstein [16] proposed the study of geometric optics for certain nonlinear equations, including (1.1). In the same paper, Weinstein proves that on the sphere $S^{2}$, the wave equation with a focussing cubic nonlinearity has a family of solutions $u^{\epsilon}$ with $\left\|u^{\epsilon}\right\|_{L^{2}}=\epsilon$, and concentrating around geodesics as $\epsilon \rightarrow 0$. The proof relies on some estimates established by Stanton and Weinstein [15].

Later work of Floer and Weinstein [5] showed that, if $X_{0}$ is any nondegenerate critical point of $V$, then for all sufficiently small $\epsilon$ there exists a standing wave solution $u^{\epsilon}$ of (1.1) such that $u^{\epsilon}$ concentrates around $X_{0}$ as $\epsilon \rightarrow 0$. This was

Received January 14, 2000. 
subsequently generalized in several papers of Y.-G. Oh, see for example [13], [14]. Recent work on the same problem includes papers of Ambrosetti et al [1] and $\mathrm{Li}[12]$.

The papers mentioned above are all concerned with standing wave solutions, and thus reduce to the study of elliptic equations. A first result about the full dynamic problem (1.1) was established by Grillakis, Shatah, and Strauss [6]. They establish, as a special case of a more general theory, the orbital stability of the standing wave solutions of Floer and Weinstein when the critical point of $V$ is a local minimum.

There has also been a good deal of work in the mathematical physics community on this and related questions, often in the general framework of studying nonintegrable perturbations of integrable systems. For example, problems very similar to the ones we consider here are studied in Kaup and Newell [9] and Keener and McLaughlin [10].

The present paper is the first that we know of to establish any rigorous results about the asymptotic behavior of (1.1) in the case where the limiting classical trajectory is nontrivial. We do not linearize the equation. We show instead that the result follows easily from conservation laws for the equation, if the mass density of the solution can be shown to be sufficiently close to a point mass. We accomplish the latter using well-known results on the nonlinear stability of ground states. Many of the techniques we use were developed in [4] and [8] in somewhat different contexts.

\section{Preliminaries}

For two vectors $u, v \in \mathbb{C}$, we use "." to denote the real inner product, $u \cdot v=$ $\frac{1}{2}(u \bar{v}+\bar{u} v)$.

We write $o_{\epsilon}(1)$ to denote a quantity that vanishes as $\epsilon \rightarrow 0$.

We follow the convention that repeated indices are summed.

We also assume that the exponent $p$ in (1.1) satisfies $1<p<1+\frac{4}{n}$.

2.1. Densities. We define a number of functionals acting on functions $v \in$ $H^{1}\left(\mathbb{R}^{n}\right)$ : the total mass

$$
M^{\epsilon}[v]=\int m_{v}^{\epsilon} d x, \quad m_{v}^{\epsilon}=\frac{1}{\epsilon^{n}}|v|^{2}
$$

the total energy

$$
E^{\epsilon}[v]=\int e_{v}^{\epsilon} d x, \quad e_{v}^{\epsilon}=\frac{1}{2 \epsilon^{n-2}}|D v|^{2}-\frac{2}{\epsilon^{n}} \frac{|v|^{p+1}}{p+1}+\frac{1}{\epsilon^{n}} V(x)|v|^{2} ;
$$

and the momentum

$$
P^{\epsilon}[v]=\int p_{v}^{\epsilon} d x, \quad p_{v}^{\epsilon}=\frac{1}{\epsilon^{n-1}} i v \cdot D v .
$$

In the final equation, $p_{v}^{\epsilon}$ is a vector with components $p_{v}^{\epsilon, j}=\frac{1}{\epsilon^{n-1}} i v \cdot v_{x_{j}}$, for $j=1, \ldots, n$. 
Smooth solutions of (1.1) satisfy the following identities.

$$
\begin{gathered}
\frac{\partial}{\partial t} m_{u^{\epsilon}}^{\epsilon}=-\operatorname{div} p_{u^{\epsilon}}^{\epsilon}, \\
\frac{\partial}{\partial t} e_{u^{\epsilon}}^{\epsilon}=\frac{1}{\epsilon^{n-2}} \operatorname{div}\left(D u^{\epsilon} \cdot u_{t}^{\epsilon}\right),
\end{gathered}
$$

and

$$
\frac{\partial}{\partial t} p_{u^{\epsilon}}^{\epsilon, j}=-\frac{1}{\epsilon^{n-2}}\left(u_{x_{j}}^{\epsilon} \cdot u_{x_{k}}^{\epsilon}\right)_{x_{k}}+\left(e_{u^{\epsilon}}^{\epsilon}+\frac{1}{\epsilon^{n-1}} i u^{\epsilon} \cdot u_{t}^{\epsilon}\right)_{x_{j}}-V_{x_{j}}(x) m_{u^{\epsilon}}^{\epsilon}
$$

For a function $v$ taking values in $\mathbb{C}$,

$$
\begin{aligned}
\left|v_{x_{j}}\right|^{2} & =\left(v_{x_{j}} \cdot \frac{v}{|v|}\right)^{2}+\left(v_{x_{j}} \cdot \frac{i v}{|v|}\right)^{2} \\
& =\left(|v|_{x_{j}}\right)^{2}+\epsilon^{n-2} \frac{\left|p_{v}^{\epsilon, j}\right|^{2}}{m_{v}^{\epsilon}} .
\end{aligned}
$$

Using this fact, we can decompose the energy in the form $E^{\epsilon}[v]=E_{b}^{\epsilon}[v]+E_{k}^{\epsilon}[v]+$ $E_{p}^{\epsilon}[v]$, where

$$
\begin{gathered}
E_{b}^{\epsilon}[v]=\int e_{b, v}^{\epsilon}, \quad e_{b, v}^{\epsilon}=\frac{2}{\epsilon^{n}}|D| v||^{2}-\frac{1}{2 \epsilon^{n}} \frac{|v|^{p+1}}{p+1} ; \\
E_{k}^{\epsilon}[v]=\int e_{k, v}^{\epsilon}, \quad e_{k, v}^{\epsilon}=\frac{\left|p_{v}^{\epsilon}\right|^{2}}{2 m_{v}^{\epsilon}} ; \\
E_{p}^{\epsilon}[v]=\int e_{p, v}^{\epsilon}, \quad e_{p, v}^{\epsilon}=\frac{1}{\epsilon^{n}} V(x)|v|^{2}=V m_{v}^{\epsilon} .
\end{gathered}
$$

We refer to these as the binding energy, the kinetic energy, and the potential energy respectively. Note that the binding enery depends only on the magnitude $|v|$ of $v$. Although the total energy $E^{\epsilon}$ is conserved for solutions of (1.1), in general $E_{b}^{\epsilon}, E_{k}^{\epsilon}$, and $E_{p}^{\epsilon}$ can vary with time.

2.2. Existence of solution. It is known that for initial data $\phi^{\epsilon} \in H^{1}\left(\mathbb{R}^{n}\right)$, (1.1) has a unique solution that exists globally in time, and that these solutions depend continuously on the initial data; see [3], Theorem 6.3.2 and Remark 6.3.4.

If in addition $\phi^{\epsilon} \in H^{2}\left(\mathbb{R}^{n}\right)$ then $u^{\epsilon}(t) \in H^{2}\left(\mathbb{R}^{n}\right)$ for all $t>0$; see [3], Theorem 5.2.3 and Remark 5.2.9.

As a consequence, if $\phi^{\epsilon} \in H^{2}\left(\mathbb{R}^{n}\right)$ then the flux terms in (2.4), (2.5), and (2.6) integrate to zero, and thus

$$
\begin{gathered}
\frac{d}{d t} M^{\epsilon}\left[u^{\epsilon}(t)\right]=\frac{d}{d t} E^{\epsilon}\left[u^{\epsilon}(t)\right]=0, \\
\frac{d}{d t} P^{\epsilon, j}\left[u^{\epsilon}(t)\right]=-\int_{\mathbb{R}^{n}} V_{x_{j}}(x) m_{u^{\epsilon}}^{\epsilon} d x .
\end{gathered}
$$


These identities remain valid for initial data $\phi^{\epsilon} \in H^{1}$ by an approximation argument and the continuous dependence on the data.

We remark that the proof of well-posedness makes use of the estimate

$$
\left\|D u^{\epsilon}(t)\right\|_{L^{2}} \leq C_{\epsilon}
$$

for all $t>0$, where the constant $C_{\epsilon}$ depends on the $H^{1}$ norm of the intial data $\phi^{\epsilon}$. We briefly recall why this holds. We find from the conservation of mass that

$$
E_{b}^{\epsilon}\left[u^{\epsilon}(t)\right]+E_{k}^{\epsilon}\left[u^{\epsilon}(t)\right]=E^{\epsilon}\left[u^{\epsilon}(t)\right]-E_{p}^{\epsilon}\left[u^{\epsilon}(t)\right] \leq C,
$$

using (2.8) and the assumption that $V$ is bounded below. Once this is known, one can use the Gagliardo-Nirenberg inequality

$$
\|v\|_{L^{q}} \leq C\|v\|_{L^{2}}^{\theta}\|D v\|_{L^{2}}^{1-\theta}, \quad \text { where } \frac{n}{q}=\theta \frac{n}{2}+(1-\theta)\left(\frac{n}{2}-1\right),
$$

to estimate $\left\|u^{\epsilon}(t)\right\|_{p+1} \leq C$. At this stage we need to use the assumption $p<1+\frac{4}{n}$. The constant $C$ depends on $M^{\epsilon}\left[u^{\epsilon}(0)\right]$ and $\sup _{t}\left(E_{p}^{\epsilon}\left[u^{\epsilon}(t)\right]+E_{k}^{\epsilon}\left[u^{\epsilon}(t)\right]\right)$. The estimate $\left\|u^{\epsilon}(t)\right\|_{p+1} \leq C$ and (2.11) immediately imply (2.10).

2.3. Spectral estimate. For every $M>0$ we define

$$
I_{M}:=\inf \left\{E_{b}^{e}[v]: v \in H^{1}\left(\mathbb{R}^{n} ; \mathbb{R}\right), M^{\epsilon}[v]=M\right\} .
$$

Note that, by scale invariance, $I_{M}$ does not depend of $\epsilon$.

It is known that there is a positive, radial function $s^{\epsilon}$ such that

$$
M^{\epsilon}\left[s^{\epsilon}\right]=M, \quad E_{b}^{\epsilon}\left[s^{\epsilon}\right]=I_{M} .
$$

This minimizer is unique in the sense that if $\phi$ is any other function such that $M^{\epsilon}[\phi]=M$ and $E_{b}^{\epsilon}[\phi]=I_{M}$, then $\phi=\tau_{y} s^{\epsilon}$ for some $y \in \mathbb{R}^{n}$. Here $\tau_{y} f$ denotes $f$ translated by $y$, that is, $\tau_{y} f(x)=f(x-y)$.

In fact more is true:

Proposition 1. There exist constants $C, h>0$ (depending on $M, n, p)$ such that

$$
\begin{aligned}
\min _{y \in \mathbb{R}^{n}}\left\{\int \frac{1}{\epsilon^{n}}\left(\phi-\tau_{y} s^{\epsilon}\right)^{2}+\int \frac{1}{\epsilon^{n-2}}\left|D\left(\phi-\tau_{y} s^{\epsilon}\right)\right|^{2}\right\} & \leq \\
& C\left(E_{b}^{\epsilon}(\phi)-E_{b}^{\epsilon}\left(s^{\epsilon}\right)\right) .
\end{aligned}
$$

for all nonnegative $\phi \in H^{1}\left(\mathbb{R}^{n}\right)$ such that $M^{\epsilon}(\phi)=M$ and $E_{b}^{\epsilon}(\phi)-E_{b}^{\epsilon}\left(s^{\epsilon}\right)<h$.

This was proven by Michael Weinstein [17], [18] in 1 and 3 space dimensions. Estimates in a subsequent paper of Kwong [11] combined with Weinstein's arguments show that Proposition 1 is valid without restrictions on the dimension.

This is the only place we use any specific properties of the nonlinearity, and indeed our results remain valid if the nonlinearity $|u|^{p-1} u$ is replaced by any $f(u)=F^{\prime}(u)$ such that an estimate like (2.12) holds when $\frac{1}{p+1}|u|^{p+1}$ is replaced by $F(u)$ in the binding energy. 
Once uniqueness is granted, it is easy to see that $s^{\epsilon}(x)=s^{1}\left(\frac{x}{\epsilon}\right)$. It is known that there are constants $\alpha, C>0$ such that

$$
s^{\epsilon}(|x|) \leq C e^{-\alpha|x| / \epsilon}
$$

for all $x$.

2.4. A weak norm. We let $C^{1}\left(\mathbb{R}^{n}\right)$ denote the space of continuously differentiable, globally bounded and Lipschitz functions on $\mathbb{R}^{n}$, with norm

$$
\|\phi\|_{C^{1}}:=\|\phi\|_{\infty}+\|D \phi\|_{\infty} .
$$

We write $C^{1 *}$ to denote the corresponding dual space, with the dual norm defined in the standard way. We identify finite Radon measures $\mu$ on $\mathbb{R}^{n}$ with elements of $C^{1 *}$ in the natural way, so that

$$
\|\mu\|_{C^{1 *}}=\sup \left\{\int_{\mathbb{R}^{n}} \phi(x) \mu(d x): \phi \in C^{1}\left(\mathbb{R}^{n}\right),\|\phi\|_{C^{1}} \leq 1\right\} .
$$

Also, given an integrable function $f \in L^{1}\left(\mathbb{R}^{n}\right)$, we write $f d x$ to denote the Radon measure whose density with respect to Lebesgue measure is $f$.

The $C^{1 *}$ norm is of course somewhat weaker than the standard norms on measures or $L^{1}$ functions. It is closely related to the length of the minimal connection, as defined in Brezis, Coron, and Lieb [2]. For example, it is not hard to verify that for any $\xi, \eta \in \mathbb{R}^{n}$,

$$
\left\|\delta_{\xi}-\delta_{\eta}\right\|_{C^{1 *}}=\frac{2|\xi-\eta|}{2+|\xi-\eta|} .
$$

To see this, suppose $\xi, \eta \in \mathbb{R}^{n}$ are given, and consider any $f \in C^{1}\left(\mathbb{R}^{n}\right)$. Then for any $\theta \in[0,1]$,

$$
\begin{aligned}
\int f\left(\delta_{\xi}-\delta_{\eta}\right)=f(\xi)-f(\eta) \leq(1-\theta) \mid & f(\xi)-f(\eta)|+\theta| f(\xi)-f(\eta) \mid \\
& \leq(1-\theta) 2\|f\|_{\infty}+\theta\|D f\|_{\infty}|\xi-\eta| .
\end{aligned}
$$

Selecting $\theta=\frac{2}{2+|\xi-\eta|}$ yields that $\left\|\delta_{\xi}-\delta_{\eta}\right\|_{C^{1 *}} \leq \frac{2|\xi-\eta|}{2+|\xi-\eta|}$. On the other hand, defining $f(x):=\theta \max \left\{\frac{1}{2}|\xi-\eta|-|\xi-x|,-\frac{1}{2}|\xi-\eta|\right\}$, for $\theta$ as above, it is easy to check that $\|f\|_{C^{1}}=1$ and $f(\xi)-f(\eta)=\frac{2|\xi-\eta|}{2+|\xi-\eta|}$.

Note that (2.14) implies that for any $K>0$ there exists some constant $C=$ $C(K)$ such that

$$
|\xi-\eta| \leq C\left\|\delta_{\xi}-\delta_{\eta}\right\|_{C^{1 *}} \quad \text { whenever } \quad\left\|\delta_{\xi}-\delta_{\eta}\right\|_{C^{1 *}} \leq K .
$$

2.5. Statement of result. We assume throughout this paper that $V: \mathbb{R}^{n} \rightarrow \mathbb{R}$ is $C^{2}$, and that $\|V\|_{C^{2}}$ is finite. This assumption could be weakened at the expense of complicating the arguments a little.

We assume there are $X_{0}, P_{0} \in \mathbb{R}^{n}$ and $M>0$ such that

$$
\begin{gathered}
M^{\epsilon}\left(\phi^{\epsilon}\right)=M \quad \text { for all } \epsilon \in(0,1] ; \\
P^{\epsilon}\left(\phi^{\epsilon}\right) \rightarrow P_{0} ;
\end{gathered}
$$


and

$$
\left\|m_{\phi^{\epsilon}}^{\epsilon} d x-M \delta_{X_{0}}\right\|_{C^{1 *}} \rightarrow 0 \quad \text { as } \epsilon \rightarrow 0 .
$$

Informally, this amounts to assuming that $\phi^{\epsilon}$ looks like a point particle with total mass $M$ and momentum $P_{0}$ located at the point $X_{0}$. We also assume that

$$
E^{\epsilon}\left(\phi^{\epsilon}\right) \rightarrow I_{M}+\frac{1}{2 M}\left|P_{0}\right|^{2}+M V\left(X_{0}\right) \quad \text { as } \epsilon \rightarrow 0 .
$$

In view of Proposition 1 and Lemma 1, assumptions (2.16) - (2.18) imply that $\lim \inf E^{\epsilon}\left(\phi^{\epsilon}\right) \geq I_{M}+\frac{1}{2 M}\left|P_{0}\right|^{2}+M V\left(X_{0}\right)$, so the final assumption (2.19) essentially means that the energy of $\phi^{\epsilon}$ is asymptotically as small as possible, given the constraints imposed by $(2.16)-(2.18)$.

Note that initial data satisfying the above assumptions exist; for example, one could take

$$
\phi^{\epsilon}(x)=s^{\epsilon}\left(x-X_{0}\right) e^{i \frac{V_{0} \cdot x}{\epsilon}}, \quad V_{0}=\frac{P_{0}}{M} .
$$

Henceforth, $u^{\epsilon}$ denotes a solution to (1.1) with initial data $\phi^{\epsilon}$ satisfying the above assumptions.

We will write $m^{\epsilon}, p^{\epsilon}$, instead of $m_{u^{\epsilon}}^{\epsilon}, p_{u^{\epsilon}}^{\epsilon}$, and so on, and similarly $P^{\epsilon}(t)$, $E_{k}^{\epsilon}(t)$ instead of $P^{\epsilon}\left[u^{\epsilon}(t)\right], E_{k}^{\epsilon}\left[u^{\epsilon}(t)\right]$ etc.

We define $\rho^{\epsilon}:=\left|u^{\epsilon}\right|$. Since the binding energy depends $E_{b}^{\epsilon}(v)$ depends only on $|v|$, it is clear that $E_{b}^{\epsilon}\left(\rho^{\epsilon}\right)=E_{b}^{\epsilon}\left(u^{\epsilon}\right)$.

Also, we will write $X(t), P(t)$ for the classical trajectories, solving the ODE

$$
M \dot{X}=P, \quad \dot{P}=-M D V(X), \quad X(0)=X_{0}, P(0)=P_{0} .
$$

Note that

$$
\frac{|P(t)|^{2}}{2 M}+M V(X(t))=\frac{\left|P_{0}\right|^{2}}{2 M}+M V\left(X_{0}\right), \quad \forall t>0 .
$$

We will prove

Theorem 1. Suppose that $u^{\epsilon}$ is a solution of (1.1) with initial data satisfying $(2.16)-(2.19)$, and that $u^{\epsilon}$ satisfies (2.8) and (2.9). Suppose also that $X(t), P(t)$ solve (2.20).

Then

$$
\left\|m^{\epsilon}(t) d x-M \delta_{X(t)}\right\|_{C^{1 *}}+\left\|p^{\epsilon}(t) d x-P(t) \delta_{X(t)}\right\|_{C^{1 *}} \rightarrow 0 \quad \text { as } \epsilon \rightarrow 0
$$

uniformly for $t \in[0, T]$, for any $T>0$.

In addition, for $\epsilon>0$ there exist functions $y^{\epsilon}:[0, \infty) \rightarrow \mathbb{R}^{n}$ such that $y^{\epsilon}(t) \rightarrow$ $X(t)$ as $\epsilon \rightarrow 0$, uniformly for $t \in[0, T]$ for any $T>0$, and

$$
\frac{1}{\epsilon^{n}}\left\|\rho^{\epsilon}-\tau_{y^{\epsilon}} s^{\epsilon}\right\|_{L^{2}}^{2}+\frac{1}{\epsilon^{n-2}}\left\|D\left(\rho^{\epsilon}-\tau_{y^{\epsilon}} s^{\epsilon}\right)\right\|_{L^{2}}^{2} \rightarrow 0 \quad \text { as } \epsilon \rightarrow 0 .
$$

Also, $P^{\epsilon}(t) \rightarrow P(t)$ uniformly for $t \in[0, T]$, and

$$
\frac{1}{\epsilon^{n}}\left\|\epsilon \frac{i u^{\epsilon} \cdot D u^{\epsilon}}{\rho^{\epsilon}}-\frac{P^{\epsilon}(t)}{M} \rho^{\epsilon}\right\|_{L^{2}}^{2} \rightarrow 0 \quad \text { as } \epsilon \rightarrow 0 .
$$


Remark 1. Since $\left\|m^{\epsilon} d x\right\|_{C^{0 *}},\left\|p^{\epsilon} d x\right\|_{C^{0 *}}$ are uniformly bounded, (2.22) immediately implies a slightly stronger result: $m^{\epsilon}(t) d x \rightarrow M \delta_{X(t)}$ and $p^{\epsilon}(t) d x \rightarrow$ $P(t) \delta_{X(t)}$ in the weak-* topology of measures as the dual of $C^{0}$.

Remark 2. The assertion $u^{\epsilon}(x, t) \sim s^{\epsilon}\left(x-y^{\epsilon}\right) e^{i \frac{P^{\epsilon} \cdot x}{M \epsilon}}$ is made precise in (2.23) and (2.24).

\section{Proofs}

Let $\zeta \in C_{c}^{\infty}$ be a smooth function such that

$$
\zeta(x)=1 \text { if }|x| \leq R, \quad \zeta(x)=0 \text { if }|x| \geq 2 R .
$$

Here $R$ is some large number that will be chosen in the proof of Lemma 5 below. The choice of $R$ will depend only on $T$, the initial data, and the potential $V$. The choice of $R$ will imply, among other things, that $|X(t)| \leq R$ for all $t \in[0, T]$.

We define

$$
X^{\epsilon}(t)=\frac{1}{M} \int x \zeta(x) m^{\epsilon}(x) d x
$$

Define

$$
\eta^{\epsilon}(t)=\left|X^{\epsilon}(t)-X(t)\right|+\left|P^{\epsilon}(t)-P(t)\right|+\left|M V(X(t))-E_{p}(t)\right| .
$$

Theorem 1 will follow from a simple argument involving Grönwall's inequality, and the following

Proposition 2. There exist positive constants $C, h_{0}$ and $\epsilon_{0}$ such that if

$$
T_{\epsilon}^{*}:=\sup \left\{t \in[0, T]: \eta^{\epsilon}(s)<h_{0} \quad \forall s \in(0, t)\right\} .
$$

then

$$
\left\|m^{\epsilon}(t) d x-M \delta_{X(t)}\right\|_{C^{1}\left(\mathbb{R}^{n}\right)^{*}}+\left\|p^{\epsilon}(t) d x-P^{\epsilon}(t) \delta_{X(t)}\right\|_{C^{1}\left(\mathbb{R}^{n}\right)^{*}} \leq C \eta^{\epsilon}(t)+o_{\epsilon}(1)
$$

whenever $t \leq T_{\epsilon}^{*}$ and $0<\epsilon<\epsilon_{0}$.

We first assume Proposition 2, as well as several other lemmas which will be established below, and present the proof of our main result.

Proof of Theorem 1. 1. We claim that to prove Theorem 1 it suffices to show that

$$
\frac{d \eta^{\epsilon}}{d t} \leq C \eta^{\epsilon}(t)+o_{\epsilon}(1)
$$

for all $t \leq T_{\epsilon}^{*}$. Indeed, the assumptions on the initial data imply that $\eta^{\epsilon}(0)=$ $o_{\epsilon}(1)$, so that $(3.1)$ readily implies that

$$
\eta^{\epsilon}(t) \leq o_{\epsilon}(1) e^{c t}
$$

for all $t<T_{\epsilon}^{*}$. In view of the definition of $T_{\epsilon}^{*}$ and the (obvious) continuiuty of $\eta^{\epsilon}$, this implies that $T_{\epsilon}^{*}=T$ for all sufficiently small $\epsilon$, and thus that $\eta^{\epsilon}(t) \rightarrow 0$ uniformly for $t \in[0, T]$. Then (2.22) follows immediately from Proposition 2. 
Once we know that $\eta^{\epsilon}(t)$ vanishes, the existence of $y^{\epsilon}(t)$ satisfying (2.23) follows from Lemma 2 and Proposition 1, and Lemma 5 implies that $y^{\epsilon}(t) \rightarrow X$ as $\epsilon \rightarrow 0$. Finally, (2.24) follows from Lemmas 1 and 2, both proven below.

2. So we estimate $\dot{\eta}^{\epsilon}$. Note that

$$
\dot{\eta}^{\epsilon} \leq\left|\dot{X}^{\epsilon}-\dot{X}\right|+\left|\dot{P}^{\epsilon}-\dot{P}\right|+\left|M D V(X) \cdot \dot{X}-\dot{E}_{p}\right| .
$$

First, we compute the derivative of the $i$ th component $X^{\epsilon, i}$ of $X^{\epsilon}(t)$ :

$$
\begin{aligned}
\dot{X}^{\epsilon, i} & =\frac{1}{M} \int x_{i} \zeta(x) m_{t}^{\epsilon}(x, t) d x \\
& =-\frac{1}{M} \int x_{i} \zeta(x) \operatorname{div} p^{\epsilon}(x, t) d x \\
& =\frac{1}{M} \int\left(\delta_{i j} \zeta(x)+x_{i} \zeta_{x_{j}}(x)\right) p^{\epsilon, j}(x, t) d x \\
& =\frac{1}{M} \int\left(\delta_{i j} \zeta(x)+x_{i} \zeta_{x_{j}}(x)\right) P^{\epsilon, j}(t) \delta_{X(t)}+C \eta^{\epsilon}(t)+o_{\epsilon}(1),
\end{aligned}
$$

using Proposition 2 and the fact that $\left\|\delta_{i j} \zeta+x_{i} \zeta_{x_{j}}\right\|_{C^{1}}<\infty$. The integral then simplifies to $P^{\epsilon, i}(t)$, in view of the definition of $\zeta$ and the fact that $|X(t)| \leq R$. Thus

$$
\left|\dot{X}^{\epsilon}-\dot{X}\right| \leq \frac{1}{M}\left|P^{\epsilon}(t)-P(t)\right|+C \eta^{\epsilon}(t)+o_{\epsilon}(1) \leq C \eta^{\epsilon}(t)+o_{\epsilon}(1) .
$$

3. Next we use (2.9), the ODE (2.20) and Proposition 2 to compute

$$
\begin{aligned}
\left|\dot{P}^{\epsilon}(t)-\dot{P}(t)\right| & =\left|-\int D V m^{\epsilon}(t) d x+M D V(X(t))\right| \\
& \leq\|D V\|_{C^{1}}\left\|m^{\epsilon}(t) d x-M \delta_{X(t)}\right\|_{C^{1 *}} \\
& \leq C \eta^{\epsilon}(t)+o_{\epsilon}(1) .
\end{aligned}
$$

4. Finally,

$$
\dot{E}_{p}^{\epsilon}(t)=\int V(x) m_{t}^{\epsilon} d x=-\int V(x) \operatorname{div} p^{\epsilon} d x=\int D V \cdot p^{\epsilon}(x) d x,
$$

and Proposition 2 implies that

$$
\left.\mid \int D V \cdot p^{\epsilon} d x-P^{\epsilon} \cdot D V(X(t))\right) \mid \leq C \eta^{\epsilon}(t)+o_{\epsilon}(1)
$$

This completes the proof of the theorem.

In the remainder of this paper, we give the proof of Proposition 2 and several auxilliary results. We first remark that

Lemma 1. $E_{k}^{\epsilon}(t)=\int \frac{\left|p^{\epsilon}\right|^{2}}{2 m^{\epsilon}} \geq \frac{\left|P^{\epsilon}\right|^{2}}{2 M}$. 
Proof. We compute

$$
\begin{aligned}
\frac{1}{2} \int \mid \frac{p^{\epsilon}}{\sqrt{m^{\epsilon}}}-\frac{P^{\epsilon}(t)}{M} & \left.\sqrt{m^{\epsilon}}\right|^{2} \\
& =\int \frac{\left|p^{\epsilon}\right|^{2}}{2 m^{\epsilon}} d x-\frac{P^{\epsilon}(t)}{M} \cdot \int p^{\epsilon} d x+\frac{\left|P^{\epsilon}(t)\right|^{2}}{2 M^{2}} \cdot \int m^{\epsilon} d x \\
& =E_{k}^{\epsilon}(t)-\frac{\left|P^{\epsilon}(t)\right|^{2}}{2 M} .
\end{aligned}
$$

It is not hard to check that, for initial data as specified, the constant $C_{\epsilon}$ in the estimate (2.10) may be taken to be $C \epsilon^{n-2}$ for some fixed $C$. Then (2.7) and (2.10) imply that $E_{k}^{\epsilon}(t) \leq \epsilon^{2-n}\left\|D u^{\epsilon}\right\|_{L^{2}}^{2} \leq C$, where $C$ is independent of $\epsilon$. Lemma 1 then implies that

$$
\left|P^{\epsilon}(t)\right| \leq C
$$

for all $t>0$.

Next we prove a lemma showing that certain quantities can be estimated solely in terms of $\eta$. This lemma uses only the fact that energy is conserved for both the PDE (1.1) and the limiting ODE (2.20).

Lemma 2. There exists some constant $C>0$ such that

$$
E_{b}^{\epsilon}(t)-E_{b}^{\epsilon}\left(s^{\epsilon}\right) \leq C \eta^{\epsilon}+o_{\epsilon}(1),
$$

and

$$
E_{k}^{\epsilon}(t)-\frac{\left|P^{\epsilon}(t)\right|^{2}}{2 M} \leq C \eta^{\epsilon}+o_{\epsilon}(1) .
$$

Proof. By the assumptions on the initial data and conservation of energy,

$$
\begin{aligned}
E_{b}^{\epsilon}(t)+E_{k}^{\epsilon}(t) & =E^{\epsilon}(t)-E_{p}^{\epsilon}(t) \\
& =E^{\epsilon}(0)-E_{p}^{\epsilon}(t) \\
& =E_{b}^{\epsilon}\left(s^{\epsilon}\right)+\frac{1}{2 M}\left|P_{0}\right|^{2}+M V\left(X_{0}\right)-E_{p}(t)+o_{\epsilon}(1) \quad \text { by }(2.19) \\
& =E_{b}^{\epsilon}\left(s^{\epsilon}\right)+\frac{1}{2 M}|P(t)|^{2}+M V(X(t))-E_{p}(t)+o_{\epsilon}(1) \quad \text { by }(2.21) \\
& \leq E_{b}^{\epsilon}\left(s^{\epsilon}\right)+\frac{1}{2 M}\left(\left|P^{\epsilon}(t)\right|^{2}+|P(t)|^{2}-\left|P^{\epsilon}(t)\right|^{2}\right)+\eta(t)+o_{\epsilon}(1) .
\end{aligned}
$$

In the last line we have used the definition of $\eta$. Note also that

$$
\begin{aligned}
\frac{1}{2 M}\left(|P(t)|^{2}-\left|P^{\epsilon}(t)\right|^{2}\right) & =\frac{1}{2 M}\left(P(t)+P^{\epsilon}(t)\right) \cdot\left(P(t)-P^{\epsilon}(t)\right) \\
& \leq C \eta(t)
\end{aligned}
$$


using (3.3). Thus

$$
\left(E_{b}^{\epsilon}(t)-E_{b}^{\epsilon}\left(s^{\epsilon}\right)\right)+\left(E_{k}^{\epsilon}(t)-\frac{\left|P^{\epsilon}(t)\right|^{2}}{2 M}\right) \leq C \eta+o_{\epsilon}(1) .
$$

Since both terms in parentheses on the left-hand side are nonnegative, (3.4) and (3.5) follow.

The next two lemmas show that if $\eta^{\epsilon}(t)$ is small, then $m^{\epsilon}(t)$ is approximately a point mass $M \delta_{y^{\epsilon}(t)}$ in an appropriate weak norm, for some point $y^{\epsilon}(t)$, and similarly the momentum density $p^{\epsilon}(t)$ is roughly the (vector) point mass $P^{\epsilon}(t) \delta_{y^{\epsilon}(t)}$. The proof of Proposition 2 will be completed when we prove later that $\left|y^{\epsilon}(t)-X(t)\right| \leq C \eta^{\epsilon}(t)+o_{\epsilon}(1)$.

The first proof relies mainly on Proposition 1.

Lemma 3. There exist constants $C, h_{0}>0$ independent of $\epsilon \in(0,1]$ such that, if $\eta^{\epsilon}(t)<h_{0}$ then there exists some point $y^{\epsilon}(t) \in \mathbb{R}^{n}$ such that

$$
\left\|m^{\epsilon}(t) d x-M \delta_{y^{\epsilon}(t)}\right\|_{C^{1}\left(\mathbb{R}^{n}\right)^{*}} \leq C \eta^{\epsilon}(t)+o_{\epsilon}(1) .
$$

Proof. 1. Let $h_{0}=\frac{h}{C}$, where $h$ is the constant from Proposition 1 and $C$ is the constant from Lemma 2. Fix any $t>0$. Recall that we are writing $\rho^{\epsilon}=\left|u^{\epsilon}\right|$. Note that $\rho^{\epsilon} \in H^{1}, \rho^{\epsilon}$ is a nonnegative function, and $M^{\epsilon}\left[\rho^{\epsilon}\right]=M$. According to Lemma 2, if $\eta^{\epsilon}(t)<h_{0}$ then $E_{b}^{\epsilon}\left(\rho^{\epsilon}(t)\right)-E_{b}^{\epsilon}\left(s^{\epsilon}\right) \leq C \eta \leq C h_{0} \leq h$. Then Proposition 1 implies that there exists some $y^{\epsilon}(t) \in \mathbb{R}^{n}$ such that

$$
\frac{1}{\epsilon^{n}} \int\left(\rho^{\epsilon}(t)-\tau_{y^{\epsilon}} s^{\epsilon}\right)^{2} d x \leq C\left(E_{b}^{\epsilon}\left(\rho^{\epsilon}(t)\right)-E_{b}^{\epsilon}\left(s^{\epsilon}\right)\right) \leq C \eta^{\epsilon}(t) .
$$

In the rest of this proof of this lemma we will assume for notational simplicity that $y^{\epsilon}(t)=0$, and we will write $s^{\epsilon}$ instead of $\tau_{y^{\epsilon}} s^{\epsilon}$. This does not involve any loss of generality.

To prove the lemma, we now need to show that

$$
\left|\int_{\mathbb{R}^{n}} \psi m^{\epsilon} d x-M \psi(0)\right| \leq C \eta^{\epsilon}(t)+o_{\epsilon}(1) \quad \text { as } \epsilon \rightarrow 0 .
$$

for all $\psi \in C^{1}\left(\mathbb{R}^{n}\right)$ such that $\|\psi\|_{C^{1}} \leq 1$. For any such $\psi$,

$$
\begin{aligned}
\int \psi(x) m_{s^{\epsilon}}^{\epsilon}(d x)-M \psi(0) & =\frac{1}{\epsilon^{n}} \int(\psi(x)-\psi(0))\left(s^{\epsilon}(x)\right)^{2}(d x) \\
& \leq \frac{1}{\epsilon^{n}} \int|x|\left(s^{1}\left(\frac{x}{\epsilon}\right)\right)^{2} d x \\
& =C \epsilon,
\end{aligned}
$$

after a change of variables. So it suffices to show that

$$
\left|\int_{\mathbb{R}^{n}} \psi m^{\epsilon} d x-\int_{\mathbb{R}^{n}} \psi m_{s^{\epsilon}}^{\epsilon} d x\right|=\left|\frac{1}{\epsilon^{n}} \int_{\mathbb{R}^{n}} \tilde{\psi}\left(\left(\rho^{\epsilon}\right)^{2}-\left(s^{\epsilon}\right)^{2}\right)(d x)\right| \leq C \eta^{\epsilon}(t)+o_{\epsilon}(1),
$$

for $\tilde{\psi}(x):=\psi(x)-\psi(0)$. 
2. The elementary inequality $a^{2}-b^{2} \leq 2(a-b)^{2}+b^{2}$ and (3.7) imply that

$$
\frac{1}{\epsilon^{n}} \int_{\mathbb{R}^{n} \backslash B_{r}} \tilde{\psi}\left(\left(\rho^{\epsilon}\right)^{2}-\left(s^{\epsilon}\right)^{2}\right) d x \leq C\|\tilde{\psi}\|_{L^{\infty}}\left(\eta^{\epsilon}(t)+F(r / \epsilon)\right),
$$

where

$$
F(R):=\frac{1}{\epsilon^{n}} \int_{\mathbb{R}^{n} \backslash B_{\epsilon R}(0)}\left(s^{\epsilon}\right)^{2} d x .
$$

Note also that $F$ is independent of $\epsilon$ since, as remarked earlier, $s^{\epsilon}(x)=s^{1}\left(\frac{x}{\epsilon}\right)$. One easily verifies from (2.13) that

$$
F(r) \leq C e^{-\tilde{\alpha} r}
$$

for some $C, \tilde{\alpha}>0$.

Also, since $\|\psi\|_{C^{1}} \leq 1,|\tilde{\psi}(x)|=|\psi(x)-\psi(0)| \leq|x|$, so

$$
\frac{1}{\epsilon^{n}} \int_{B_{r}} \tilde{\psi}\left(\left(\rho^{\epsilon}\right)^{2}-\left(s^{\epsilon}\right)^{2}\right) d x \leq \frac{r}{\epsilon^{n}} \int_{\mathbb{R}^{n}}\left(\left(\rho^{\epsilon}\right)^{2}+\left(s^{\epsilon}\right)^{2}\right) d x=2 M r .
$$

Combining this with (3.8) and noting that $\|\tilde{\psi}\|_{L^{\infty}} \leq 2\|\psi\|_{L^{\infty}} \leq 2$, we find that

$$
\frac{1}{\epsilon^{n}} \int_{\mathbb{R}^{n}} \tilde{\psi}\left(\left(\rho^{\epsilon}\right)^{2}-\left(s^{\epsilon}\right)^{2}\right) d x \leq C\left(\eta^{\epsilon}(t)+r+F(r / \epsilon)\right) .
$$

In view of (3.9), the result follows by selecting $r=\epsilon \log \epsilon$.

The argument of the next proof is very similar to the preceeding one, except that here we use Lemma 1 to control the momentum, whereas before we used Proposition 1 to control the mass.

Lemma 4. There exists a constant $C>0$, independent of $\epsilon$, such that if $\eta^{\epsilon}(t)<$ $h_{0}$ then

$$
\left\|p^{\epsilon}(t) d x-P^{\epsilon}(t) \delta_{y^{\epsilon}(t)}\right\|_{C^{1}\left(\mathbb{R}^{n}\right)^{*}} \leq C \eta^{\epsilon}(t)+o_{\epsilon}(1) .
$$

Here $h_{0}$ is the small constant from Lemma 3.

Proof. Write $g^{\epsilon}(t):=\frac{p^{\epsilon}(t)}{\sqrt{m^{\epsilon}(t)}}-\frac{P^{\epsilon}(t)}{M} \sqrt{m^{\epsilon}(t)}$, and note that Lemmas 1 and 2 imply that

$$
\left\|g^{\epsilon}(t)\right\|_{L^{2}}^{2} \leq C \eta^{\epsilon}(t)+o_{\epsilon}(1) .
$$

Taking $\tilde{\psi}$ to equal the characteristic function of $\mathbb{R}^{n} \backslash B_{r}$ in (3.8), we obtain

$$
\int_{\mathbb{R}^{m} \backslash B_{r}} m^{\epsilon}=\frac{1}{\epsilon^{n}} \int_{\mathbb{R}^{n} \backslash B_{r}}\left(\left(\rho^{\epsilon}\right)^{2}-\left(s^{\epsilon}\right)^{2}\right) d x+F(r / \epsilon) \leq C\left(\eta^{\epsilon}(t)+F(r / \epsilon)\right) .
$$

The previous two estimates and Cauchy's inequality imply that

$$
\int_{\mathbb{R}^{n} \backslash B_{r}}\left|g^{\epsilon}\right| \sqrt{m^{\epsilon}} d x \leq C\left(\eta^{\epsilon}(t)+F(r / \epsilon)+o_{\epsilon}(1)\right)
$$


for any $r>0$. It is easy to check that $\int_{\mathbb{R}^{n}} g^{\epsilon} \sqrt{m^{\epsilon}}=0$, so we see that

$$
\left|\int_{B_{r}} g^{\epsilon} \sqrt{m^{\epsilon}} d x\right| \leq C\left(\eta^{\epsilon}(t)+F(r / \epsilon)+o_{\epsilon}(1)\right) .
$$

Thus for any $\psi \in C^{1}$ such that $\|\psi\|_{C^{1}} \leq 1$,

$$
\begin{aligned}
\left|\int_{\mathbb{R}^{n}} \psi\left(p^{\epsilon}(t)-\frac{P^{\epsilon}(t)}{M^{\epsilon}(t)} m^{\epsilon}\right) d x\right| & =\left|\int_{\mathbb{R}^{n}} \psi g^{\epsilon} \sqrt{m^{\epsilon}} d x\right| \\
& \leq \int_{\mathbb{R}^{n} \backslash B_{r}}\left|g^{\epsilon}\right| \sqrt{m^{\epsilon}}+|\psi(0)|\left|\int_{B_{r}} g^{\epsilon} \sqrt{m^{\epsilon}}\right| \\
& \quad+\sup _{y \in B_{r}}|\psi(0)-\psi(y)| \int_{B_{r}}\left|g^{\epsilon}\right| \sqrt{m^{\epsilon}} \\
& \leq C\left(\eta^{\epsilon}(t)+F(r / \epsilon)+r\right)+o_{\epsilon}(1) .
\end{aligned}
$$

Choosing $r=\epsilon \log \epsilon$, we deduce that $\left\|p^{\epsilon}(t) d x-\frac{P^{\epsilon}(t)}{M} m^{\epsilon}(t) d x\right\|_{C^{1 *}} \leq C \eta^{\epsilon}+o_{\epsilon}(1)$. Now the conclusion follows from Lemma 3 and the triangle inequality.

Combining Lemmas 3 and 4 we find that

$$
\left\|m^{\epsilon} d x-M \delta_{y^{\epsilon}(t)}\right\|_{C^{1}\left(\mathbb{R}^{n}\right)^{*}}+\left\|p^{\epsilon} d x-P^{\epsilon}(t) \delta_{y^{\epsilon}(t)}\right\|_{C^{1}\left(\mathbb{R}^{n}\right)^{*}} \leq C \eta^{\epsilon}(t)+o_{\epsilon}(1)
$$

whenever $\eta^{\epsilon}(t) \leq h_{0}$. To complete the proofs of Proposition 2 and Theorem 1 it therefore only remains to prove

Lemma 5. There exists some $\epsilon_{0}>0$ such that if $\eta^{\epsilon}(t) \leq h_{0}$ and $\epsilon<\epsilon_{0}$, then

$$
\left\|\delta_{y^{\epsilon}(t)}-\delta_{X(t)}\right\|_{C^{1 *}} \leq\left|y^{\epsilon}(t)-X(t)\right| \leq C \eta^{\epsilon}(t)+o_{\epsilon}(1)
$$

Proof. 1. The first inequality follows from (2.14), so we only need to prove the second one.

Recall that $\zeta(x) \equiv 1$ if $|x| \leq R$, where $R$ is some large constant that we have not yet chosen. So if $\left|y^{\epsilon}(t)\right| \leq R$,

$$
\begin{aligned}
& \left|X(t)-y^{\epsilon}(t)\right| \leq\left|X^{\epsilon}(t)-y^{\epsilon}(t)\right|+\eta^{\epsilon}(t) \\
& =\left|\frac{1}{M} \int x \zeta(x)\left(m^{\epsilon}(t)-M \delta_{y^{\epsilon}(t)}\right)(d x)\right|+\eta^{\epsilon}(t) \\
& \leq C\|x \zeta\|_{C^{1}}\left\|m^{\epsilon}(t) d x-M \delta_{y^{\epsilon}(t)}\right\|_{C^{1 *}}+\eta^{\epsilon}(t) \\
& \leq C \eta^{\epsilon}(t)+o_{\epsilon}(1) \text {. }
\end{aligned}
$$

It therefore suffices to show that we can choose $R$ such that $\left|y^{\epsilon}(t)\right| \leq R$ for all $t \leq T_{\epsilon}^{*}$, when $\epsilon<\epsilon_{0}$. Thus in effect we need to bound the mobility of the particle. 
2. Fix any $\psi \in C_{c}^{1}\left(\mathbb{R}^{n}\right)$ and times $t_{1}, t_{2} \in\left[0, T_{\epsilon}^{*}\right]$ such that $t_{1}<t_{2}$, and use (2.4) to calculate

$$
\begin{aligned}
\int \psi\left(m^{\epsilon}\left(t_{2}\right)-m^{\epsilon}\left(t_{1}\right)\right) d x & =\int_{\mathbb{R}^{n}} \int_{t_{1}}^{t_{2}} \psi m_{t}^{\epsilon} d x d t \\
& =\int_{\mathbb{R}^{n}} \int_{t_{1}}^{t_{2}}-\psi \operatorname{div} p^{\epsilon} d t d x \\
& =\int_{\mathbb{R}^{n}} \int_{t_{1}}^{t_{2}} D \psi \cdot p^{\epsilon} d t d x \\
& \leq\|D \psi\|_{\infty} \int_{t_{1}}^{t_{2}}\left(2 \int_{\mathbb{R}^{n}} m^{\epsilon} d x\right)^{1 / 2}\left(\int_{\mathbb{R}^{n}} \frac{\left|p^{\epsilon}\right|^{2}}{2 m^{\epsilon}} d x\right)^{1 / 2} \\
& \leq C\left|t_{2}-t_{1}\right|\|\psi\|_{C^{1}},
\end{aligned}
$$

where the constant depends on $M$ and on the uniform bound (3.3). Thus the triangle inequality and (3.6) imply that

$$
\left\|M \delta_{y^{\epsilon}\left(t_{2}\right)}-M \delta_{y^{\epsilon}\left(t_{1}\right)}\right\|_{C^{1 *}} \leq C\left(\eta^{\epsilon}\left(t_{1}\right)+\eta^{\epsilon}\left(t_{2}\right)+\left|t_{2}-t_{1}\right|+o_{\epsilon}(1)\right) .
$$

Since $t_{i} \leq T_{\epsilon}^{*} \leq T$ for $i=1,2$,

$$
C\left(\eta^{\epsilon}\left(t_{1}\right)+\eta^{\epsilon}\left(t_{2}\right)+\left|t_{2}-t_{1}\right|+o_{\epsilon}(1)\right) \leq C(2 h+T+1):=K,
$$

for $\epsilon$ small enough that $o_{\epsilon}(1) \leq 1$. It then follows from $(2.15)$ that there exists some $C=C(K)$ such that

$$
\left|y^{\epsilon}\left(t_{2}\right)-y^{\epsilon}\left(t_{1}\right)\right| \leq C(K)\left\|M \delta_{y^{\epsilon}\left(t_{2}\right)}-M \delta_{y^{\epsilon}\left(t_{1}\right)}\right\|_{C^{1 *}} \leq K C(K)
$$

Also, it is clear from the definition of $y^{\epsilon}$ and the assumption (2.18) about the initial data that $\left|y^{\epsilon}(0)-X_{0}\right| \leq 1$ for all $\epsilon$ sufficiently small, so if we define $R:=K C(K)+\left|X_{0}\right|+1$, we obtain

$$
\left|y^{\epsilon}(t)\right| \leq R
$$

for all $t \leq T_{\epsilon}^{*}$ and all small $\epsilon$, as desired.

\section{Acknowledgments}

Bronski was partially supported by NSF grant DMS-9972869, and Jerrard was partially supported by NSF grant DMS-9970273.

We are grateful to Jean Bourgain, Chris Jones and Michael Sigal for providing us with some references.

\section{References}

[1] A. Ambrosetti, M. Badiale, and S. Cingolani, Semiclassical states on nonlinear Schrödinger equations, Arch. Rational Mech. Anal. 140 (1997), 285-300.

[2] H. Brezis, J.-M. Coron, and E. Lieb, Harmonic maps with defects, Commun. Math. Phys. 107 (1986), 649-705.

[3] Th. Cazenave, An introduction to nonlinear Schrödinger equations, third ed., Textos de Metodos Matematicos, vol. 26, Instituto de Matématica, Rio de Janeiro, 1996. 
[4] J.E. Colliander and R.L. Jerrard, Vortex dynamics for the Ginzburg-Landau-Schrödinger equation, Internat. Math. Res. Notices (1998), 333-358.

[5] A. Floer and A. Weinstein, Nonspreading wave packets for the cubic Schrödinger equation with a bounded potential, J. Funct. Anal. 69 (1986), 397-408.

[6] M. Grillakis, J. Shatah, and W. Strauss, Stability theory of solitary waves in the presence of symmetry, J. Funct. Anal. 74 (1987), 160-197.

[7] V. Guillemin and S. Sternberg, Geometric asymptotics, Mathematical Surveys, No. 14, Amer. Math. Soc., Providence, R.I. 1976.

[8] R.L. Jerrard, Vortex dynamics for the Ginzburg-Landau wave equation, Calc. Var. Partial Differential Equations 9 (1999), 1-30.

[9] D.J. Kaup and A.C. Newell, Solitons as particles, oscillators, and in slowly changing media: singular perturbation theory, Proc. Roy. Soc. A 361 (1978), 413-446.

[10] J.P. Keener and D. W. McLaughlin, Solitons under perturbations, Phys. Rev. A 16 (1977), 777-790.

[11] M.K. Kwong, Uniqueness of positive solutions of $\Delta u-u+u^{p}=0$ in $\mathbf{R}^{\mathbf{n}}$, Arch. Rational Mech. Anal. 105 (1989), 243-266.

[12] Y.Y. Li, On a singularly perturbed elliptic problem, Adv. Diff. Equations 2 (1997), 955980.

[13] Y.-G. Oh, Existence of semiclassical bound states of nonlinear Schrödinger equations with potentials of class $(v)_{\alpha}$, Comm. Partial Differential Equations 13 (1988), 1499-1519.

[14] On positive multi-lump bound states of nonlinear Schrödinger equations under multiple-well potentials, Comm. Math. Phys. 131 (1990), 223-253.

[15] R.J. Stanton and A. Weinstein, On the $L^{4}$ norm of spherical harmonics, Math. Proc. Cambridge Philos. Soc. 89 (1981), 343-358.

[16] A. Weinstein, Nonlinear stabilization of quasimodes, Proc. Amer. Math. Soc. Symposium on geometry of the Laplacian (Hawaii, 1979), Proc. Sympos. Pure Math., vol. 36, pp. 301318, Amer. Math. Soc., Providence, R.I., 1980.

[17] M. Weinstein, Modulational stability of ground states of nonlinear Schrödinger equations, SIAM J. Math. Anal. 16 (1985), 472-491.

[18] _ Lyapunov stability of ground states of nonlinear dispersive evolution equations, Comm. Pure Appl. Math 39 (1986), 51-67.

Math Department, University of Illinois, Urbana, IL 61801

E-mail address: jared@math.uiuc.edu

Math Department, University of Illinois, Urbana, IL 61801

E-mail address: rjerrard@math.uiuc.edu 